\title{
Study on Circulating Tumor Cells and Various Clinical Features and Blood-Related Indexes in Patients with Renal Cancer
}

\author{
Dianbin Song1, Zhiyong Wang ${ }^{1 *}$, Xiuming Li ${ }^{1}$, Jingjing Zhan ${ }^{2}$, Qiang Chi' ${ }^{1}$, Hui Xu${ }^{1}$, Hongyang Li ${ }^{1}$, \\ Ying Liu ${ }^{1}$
}

${ }^{1}$ Department of Urology, Affiliated Hospital of Chengde Medical College, Chengde, China

${ }^{2}$ Department of Oncology, Affiliated Hospital of Chengde Medical College, Chengde, China

Email: *songdianbin123@163.com

How to cite this paper: Song, D.B., Wang, Z.Y., Li, X.M., Zhan, J.J., Chi, Q., Xu, H., Li, H.Y. and Liu, Y. (2022) Study on Circulating Tumor Cells and Various Clinical Features and Blood-Related Indexes in Patients with Renal Cancer. Surgical Science, 13, 40-45. https://doi.org/10.4236/ss.2022.131007

Received: December 15, 2021

Accepted: January 22, 2022

Published: January 25, 2022

Copyright $\odot 2022$ by author(s) and Scientific Research Publishing Inc. This work is licensed under the Creative Commons Attribution International License (CC BY 4.0).

http://creativecommons.org/licenses/by/4.0/

\begin{abstract}
Objective: To investigate the value of CTC clinical features and blood-related test indicators in renal cancer patients by detecting the number of circulating tumor cells (CTC) in patients with renal cancer. Methods: To analyze 59 patients with renal cell carcinoma (RCC) admitted to the Department of Urology, Affiliated Hospital of Chengde Medical College from May 2018 to October 2019. According to the CTC count ( $5 \mathrm{pcs} / 3.5 \mathrm{ml}$ ), they were divided into CTC positive group and CTC negative group. The age, gender, tumor location, tumor size, hematuria, CEC (circulating endothelial cells), CTC Cluster (aggregate), gene mutation, platelet (PLT), albumin (ALB), hemoglobin (HB), alkaline phosphatase (AKP), lactate dehydrogenase (LDH) were compared between the two groups of patients and the correlation of the number of CTCs. Results: There were significant differences in tumor size, CEC, and gene mutations between the two groups $(\mathrm{P}<0.05)$. The number of CTC in patients was correlated with the levels of HB, PLT and LDH $(\mathrm{P}<0.05)$. Conclusion: The number of CTC in RCC patients is correlated with some clinical features (tumor size, CEC, gene mutation) and some related test indicators (HB, PLT, $\mathrm{LDH})$, and can be combined with the above related indicators to predict the occurrence, metastasis and prognosis of renal cancer.
\end{abstract}

\section{Keywords}

Kidney Cancer, Circulating Tumor Cells, Clinical Features, Test Indicators

\section{Introduction}

Renal cell carcinoma (RCC) is a common malignant tumor of the urinary sys- 
tem, with a variety of histological types [1], accounting for $90 \%$ of all kidney tumors, accounting for about $3 \%$ of all adult malignancies [2]. In my country, RCC ranks second in urinary system malignancies, and the incidence is showing an upward trend. Although the overall survival rate of RCC treatment has continued to increase in recent years, its progression-free survival period is still less than 1 year [3]. At present, surgical resection is the main clinical practice, but the 5 -year survival rate of this treatment is only $5 \%$, and about $40 \%$ of patients will relapse or metastasize after surgery [4]. Therefore, the current important problem facing RCC is how to prevent tumor recurrence and metastasis.

Tumor metastasis is a complex process involving multiple steps and multiple factors. The first is the shedding of tumor cells from the primary tumor, and a very small number of them will metastasize to the blood, lymph nodes or distant organs, which is called tumor micrometastasis [5]. However, this kind of micro-transfer is difficult to detect with the current routine inspection methods. Therefore, early detection of micrometastasis is very important for tumor recurrence and metastasis, and can significantly improve the prognosis. Current studies believe that circulating tumor cells (CTC) are released from the primary site or metastatic site of malignant tumors and enter the peripheral circulation. Under normal circumstances, CTCs can be phagocytosed and cleared or apoptotic, but a small number of CTCs can survive, and because it has similar antigens and heredity to the primary tumor, and may colonize a certain part of the body to form new metastases. At present, CTC is considered to be an important basis for hematological metastasis of malignant tumors, and has always received clinical attention [6]. CTC falls off into the blood and may cause metastasis in patients with malignant tumors. CTC plays an important role in tumor metastasis [7]. This study explored the application value of CTC detection in renal cancer by detecting the number of CTCs in patients with renal cell carcinoma, the relationship with various clinical characteristics and various clinical blood test indicators.

\section{Materials and Methods}

\subsection{General Information}

This study conducted a retrospective study of 59 RCC patients who visited the Department of Urology from May 2018 to October 2019 in the Affiliated Hospital of Chengde Medical College (hereinafter referred to as our hospital). Among them, there were 33 males and 26 females. The tumor was located in 34 cases on the left side and 25 cases were located on the right side. The oldest age was 85 years old and the youngest age was 32 years old. This study was approved by the Ethics Committee of the Affiliated Hospital of Chengde Medical College, and all patients signed an informed consent form.

\subsection{Judgment Criteria}

Divided into CTC positive group and CTC negative group, the criteria for CTC 
positive grouping is based on domestic and foreign literature [8], the criterion for positive group is CTC $\geq 5 / 3.5 \mathrm{~mL}$, and the negative group is CTC $<5$ Pieces/3.5 mL.

\subsection{Detection Method}

Take the early morning fasting peripheral blood of 59 patients with kidney cancer in our hospital to detect the number of CTCs, and record the age, gender, tumor location, tumor size, hematuria, CEC (circulating endothelial cells), CTC Cluste of the two groups at the same time (Polymer), gene mutation and the value of platelet (PLT), albumin (ALB), hemoglobin (HB), alkaline phosphatase (AKP), lactate dehydrogenase (LDH).

\subsection{Statistical Analysis}

Use SPSS 22.0 to perform statistical analysis on the data. The measurement data are expressed as mean \pm standard deviation, which conforms to the t-test of homogeneity of variance and normal distribution. The comparison of count data adopts the chi-square test. $\mathrm{P}<0.05$ means the difference is statistically significant.

\section{Results}

1) There is no difference in the general information of patients in the CTC-positive group and CTC-negative group (Table 1).

2) The two groups of patients had differences in tumor size, CEC, and gene mutations $(\mathrm{P}<0.05)($ Table 2$)$.

3) The number of CTC is correlated with the levels of HB, PLT and LDH in patients with renal cell carcinoma $(\mathrm{P}<0.05)$ (Table 3).

Table 1. Comparison of general information of patients.

\begin{tabular}{ccccc}
\hline & CTC positive group & CTC negative group & statistics & P value \\
\hline Gender: Male/Female) & $17 / 13$ & $16 / 13$ & $\mathrm{z}=0.3207$ & 0.8061 \\
Age $(\mathrm{x} \pm \mathrm{s})$ & $57.06 \pm 1.899$ & $56.07 \pm 1.894$ & $\mathrm{t}=0.6954$ & 0.5107 \\
Location (left/right) & $18 / 12$ & $16 / 13$ & $\mathrm{z}=1.848$ & 0.0798 \\
\hline
\end{tabular}

Note: All P values are $>0.05$, the difference is not statistically significant.

Table 2. Comparison of the clinical characteristics of the two groups of patients.

\begin{tabular}{ccccc}
\hline & CTC positive group & CTC negative group & statistics & P value \\
\hline Size $(\mathrm{cm})$ & $8.391 \pm 0.5701$ & $5.260 \pm 0.3311$ & $\mathrm{t}=4.727$ & $\mathrm{z}=1.345$ \\
Hematuria (positive/negative) & $10 / 26$ & $9 / 24$ & $\mathrm{u}=58.90$ & $<0.0001$ \\
CEC & $7.000 \pm 0.9346$ & $0.4000 \pm 0.1438$ & $\mathrm{z}=1.546$ & 0.0988 \\
CTC Cluste (positive/negative) & $3 / 27$ & $0 / 19$ & $\mathrm{z}=3.981$ & $<0.0001$ \\
Gene mutation (positive/negative) & $22 / 9$ & $3 / 16$ & $\mathrm{t}=9.415$ & $<0.0001$ \\
CTC & $11.35 \pm 0.9070$ & $1.980 \pm 0.3362$ & &
\end{tabular}


Table 3. The relationship between CTC and HB, PLT, ALB, AKP, LDH in patients with kidney cancer ( $\pm \mathrm{s})$.

\begin{tabular}{ccccccc}
\hline Group & Number of cases & HB $(\mathrm{g} / \mathrm{L})$ & PLT $(\times 10 \sim / \mathrm{L})$ & ALB $(\mathrm{g} / \mathrm{L})$ & AKP $(\mathrm{U} / \mathrm{L})$ & LDH $(\mathrm{U} / \mathrm{L})$ \\
\hline CTC positive group & 30 & $113.69 \pm 3.40$ & $209.50 \pm 7.67$ & $39.50 \pm 0.86$ & $111.70 \pm 9.20$ & $189.10 \pm 10.70$ \\
CTC negative group & 29 & $135.52 \pm 3.20$ & $172.70 \pm 12.10$ & $38.60 \pm 0.87$ & $110.90 \pm 10.30$ & $170.70 \pm 7.10$ \\
Z value & & 3.045 & 3.773 & 4.713 & 4.258 & 2.867 \\
p value & & 0.0032 & 0.048 & 0.620 & 0.056 & 0.0205 \\
\hline
\end{tabular}

\section{Conclusion}

By detecting the number of CTC in patients with kidney cancer, combined with some clinical features (tumor size, CEC, gene mutation) and some blood-related test indicators (HB, PLT, LDH), it can assist in the evaluation of its efficacy and prognosis.

\section{Discussion}

A review of related literature found that $90 \%$ of cancer-related deaths were caused by tumor metastasis [9]. Therefore, if the occurrence and metastasis of tumors can be detected at an early stage, the diagnosis rate can be significantly increased, the survival time can be prolonged, and the prognosis can be improved. In 1869, Ashworth first proposed the concept of circulating tumor cells (CTC) [10]. The occurrence and metastasis of tumors can be judged by detecting CTC [11]. In recent years, with people in-depth research on malignant tumors, the value of CTC in the clinical detection and clinical application of malignant tumors has made great progress. A large number of literatures have also reported CTC in prostate cancer, breast cancer, gastric cancer and the value of clinical diagnosis and treatment of colon cancer [12] [13]. In this study, we also found that there are significant differences in some clinical characteristics of renal cell carcinoma patients in different groups, such as tumor size, CEC level, etc, indicating the impact of CTC level on renal cell carcinoma patients, so we infer that the number of CTC is also It will have a significant impact on the prognosis of renal cancer patients and has the potential to predict the prognosis of RCC patients. It also shows that certain clinical characteristics of patients may also affect the number of CTC.

Previous studies have shown [14] that patients with malignant tumors, especially those undergoing radiotherapy and chemotherapy, are prone to malnutrition due to: easily causing gastrointestinal disorders; reduced digestion and absorption; cancer cells plundering nutrients. In this study, we also found that the $\mathrm{HB}$ value of the CTC-positive group was significantly lower than that of the negative group, suggesting that this group of patients had obvious malnutrition.

Studies have found that the occurrence and metastasis of tumor cells are related to the increase of PLT [15], the main reason is that the increase in the number of PLT can accelerate tumor metastasis. In this study, the PLT of the CTC positive group was significantly higher than that of the negative group, 
suggesting that patients in the CTC positive group are beneficial to tumor occurrence and metastasis.

It has been confirmed that LDH is present in the cytoplasm of cells, and patients with malignant tumors will have an increase in LDH [16]. In this study, the LDH in the CTC-positive group of 59 renal cancer patients was significantly higher than that in the negative group, indicating that the CTC count was significantly related to the patient's LDH. On the other hand, LDH can be used as an indicator of the disease progression of renal cancer patients, and the combination of CTC count and LDH can better judge the prognosis of renal cancer.

CTC combined with some clinical features (tumor size, CEC, gene mutation) and some related test indicators (HB, PLT, LDH) can assist renal cancer patients in judging their curative effect and prognostic evaluation, so as to achieve individualized treatment. This study is a single-center study, and the amount of data is very limited. Future studies will further expand the sample size.

\section{Fund}

S \& T Program of Chengde (201904A025).

\section{Conflicts of Interest}

The authors declare no conflicts of interest regarding the publication of this paper.

\section{References}

[1] Dunnick, N.R. (2016) Renal Cell Carcinoma: Staging and Surveillance. Abdominal Radiology (NY), 41, 1079-1085. https://doi.org/10.1007/s00261-016-0692-0

[2] Znaor, A., Lortet-Tieulent, J., Laversanne, M., et al. (2015) International Variations and Trends in Renal Cell Carcinoma Incidence and Mortality. European Urology, 67, 519-530. https://doi.org/10.1016/j.eururo.2014.10.002

[3] Na, J.C., Kim, J.H., Kim, S.Y., et al. (2020) Establishment of Patient-Derived Three-Dimensional Organoid Culture in Renal Cell Carcinoma. Investigative and Clinical Urology, 61, 216-223. https://doi.org/10.4111/icu.2020.61.2.216

[4] Yang, J., Yang, L., Li, S., et al. (2020) HGF/c-Met Promote Renal Carcinoma Cancer Stem Cells Enrichment through Upregulation of Cir-CCDC66. Technology in Cancer Research \& Treatment, 19, 1-8. https://doi.org/10.1177/1533033819901114

[5] Jiang, W.F. and Zhang, H.L. (2010) Research Progress in the Detection of Circulating Tumor Cells in Peripheral Blood. Journal of Clinical Oncology, 10, 944-947.

[6] Steeg, P.S. (2006) Tumor Metastasis: Mechanistic Insights and Clinical Challenges. Nature Medicine, 12, 895-904. https://doi.org/10.1038/nm1469

[7] Maheswaran, S. and Haber, D.A. (2010) Circulating Tumor Cells: A Window into Cancer Biology and Metastasis. Current Opinion in Genetics \& Development, 20 , 96-99. https://doi.org/10.1016/j.gde.2009.12.002

[8] Reid, A.H., Attard, G., Danila, D.C., et al. (2010) Significant and Sustained Antitumor Activity in Post-Docetaxel, Castration Resistant Prostate Cancer with the CYP17 Inhibitor Abiraterone Acetate. Journal of Clinical Oncology, 28, 1489-1495. https://doi.org/10.1200/JCO.2009.24.6819 
[9] Sun, S.C. and Sun, Z.W. (2016) Choice of Laparoscopic Inguinal Hernia Surgery: TAPP vs. TEP. Journal of Laparoscopic Surgery, 21, 85-87.

[10] Ashworth, T.R. (1869) A Case of Cancer in Which Cells Similar to Those in the Tumors Were Seen in the Blood after Death. Australasian Medical Journal, 14, 146-147.

[11] Benezeder, T., Tiran, V., Treitler, A., et al. (2017) Multigene Methylation Analysis of Enriched Circulating Tumor Cells Associates with Poor Progression-Free Survival in Metastatic Breast Cancer Patients. Oncotarget, 8, 92483-92496. https://doi.org/10.18632/oncotarget.21426

[12] Politaki, E., et al. (2017) A Comparison of Three Methods for the Detection of Circulating Tumor Cells in Patients with Early and Metastatic Breast Cancer. Cellular Physiology and Biochemistry, 44, 594-606. https://doi.org/10.1159/000485115

[13] Sun, K., Sun, S., Hu, E.W., et al. (2018) Combining CK19, hMAM, SBEM to Detect Breast Cancer Peripheral Blood Circulating Tumor Cells and Analysis of Their Correlation with Molecular Typing and Clinicopathological Characteristics. Journal of Clinical and Experimental Medicine, No. 3, 270-273.

[14] von Meyenfeldt, M. (2005) Cancer-Associated Malnutrition: An Introduction. European Journal of Oncology Nursing, 9, S35-S38.

https://doi.org/10.1016/j.ejon.2005.09.001

[15] Xiao, B., et al. (2011) Correlation between Coagulation Function, Tumor Stage and Metastasis in Patients with Renal Cell Carcinoma: A Retrospective Study. Chinese Medical Journal: English Edition, 124, 1205-1208.

[16] Chen, S.Y., Feng, L. and Du, Y. (2018) The Application of Detection of Circulating Tumor Cells in Peripheral Blood in the Prognosis of Prostate Cancer Patients. Journal of Clinical and Experimental Medicine, 17, 1660-1663. 